\title{
Steroid Inhaler Therapy and Oral Manifestation Similar to Median Rhomboid Glossitis (Case Report)
}

\author{
Dwi Suhartiningtyas ${ }^{1, *}$ Syifa Nabila Farah Fauziah Nur ${ }^{2}$
}

\author{
${ }^{1}$ Lecturer at School of Dentistry, Faculty of Medicine and Health Science, Universitas Muhammadiyah Yogyakarta \\ ${ }^{2}$ Student at School of Dentistry, Faculty of Medicine and Health Science, Universitas Muhammadiyah Yogyakarta \\ *Corresponding author. Email: dwi.suhartiningtyas@umy.ac.id
}

\begin{abstract}
One of the oral manifestations in the use of a steroid inhaler is Median Rhomboid Glossitis (MRG). It is a benign condition that is asymptomatic and appears as central papillary atrophy of the tongue. This case report presents the use of a steroid inhaler in an asthma patient with manifestation in the oral cavity similar to MRG. A 27-year-old woman came to Academic Dental Hospital Universitas Muhammadiyah Yogyakarta with a complaint of discomfort on her tongue because it looked whiter than usual. The patient had an asthma attack 3 days before a complaint appeared on the tongue. The intraoral examination shows white plaque that easily rubbed without leaving the reddish area. Another finding in the middle of the tongue appears reddish areas that are similar to MRG. Treatments that have been given are instruction to brush the tongue using a tongue scraper, gargle using water shortly after the use of a steroid inhaler when the asthma attack subsides, administer the mouthwash containing povidone-iodine $1 \%$, and control 1 week later. The evaluation for 6 weeks long gave quite a good result related to white lesions diagnosed as a coated tongue, while the red area in the median dorsal tongue similar to MRG is another finding as an oral manifestation of the use of a steroid inhaler. Steroid inhaler for the treatment of asthma can trigger several lesions such as MRG. Confirmation of MRG by mycological examination is needed to ensure the presence of fungal colonization.
\end{abstract}

\section{Keywords: Asthma, Median Rhomboid Glossitis, Oral Manifestation, Steroid Inhaler}

\section{INTRODUCTION}

Median Rhomboid Glossitis (MRG), also known as central papillary atrophy, is a benign condition on the tongue, asymptomatic and characterized by atrophy of filiform papillae on the dorsal surface of the tongue in the shape of a rhomboid [1][2]. The clinical appearance of the lesions varies. They may be raised, fissured, or lobed and sometimes show a darker color. There is also a flat, dark pink, bald (atrophic) appearance on the median dorsum of the tongue with a white coating on the outer side. It is estimated that the prevalence of MRG is around $0.01-1.0 \%$ of the population [3].

Median Rhomboid Glossitis was initially considered a developmental defect that occurs during the embryogenesis process due to the failure of the tubercular impart. The current theory believes that MRG is a chronic infection caused by Candida albicans [4][5]. Several related factors that predispose to MRG include smoking, use of dentures, diabetes mellitus, use of steroid inhalers in an asthma patient and chronic lung disease such as Chronic Obstructive Pulmonary Disease (COPD), as well as the use of broad-spectrum antimicrobials [6][7][8].

Asthma is a non-communicable disease characterized by repeated attacks of shortness of breath and wheezing, with different severity and frequency for each individual [10]. Steroid inhalers are drugs commonly used in asthma and COPD [11]. Only $10-20 \%$ of the drug dose reaches the lungs while the rest remains in the oral cavity and oropharynx. High steroid doses and a long time in the oral cavity will affect the normal flora balance. If this condition is allowed, it can lead to yeast infection. A steroid inhaler in the oral cavity can inhibit the flow of saliva so that the glucose concentration in saliva is higher. As a result, this condition will encourage the growth and proliferation of Candida [12][13]. This case report presents the use of a steroid inhaler in an asthma patient with manifestation in the oral cavity, similar to MRG.

\section{CASE REPORT}

On October $2^{\text {nd }} 2019$, a 27-year-old woman came to Academic Dental Hospital Universitas Muhammadiyah Yogyakarta with a complaint of discomfort on the tongue because it looks whiter than usual the center of the tongue is reddish. The complaint has been felt since 3 days ago, but there is no pain or itching. The complaint has never been treated or examined by a dentist. The clinical history revealed that 3 days before the complaint appeared on the tongue, the patient had an asthma attack, and to overcome the attack, she used a steroid inhaler. During these 2 years, the patient routinely used a steroid inhaler at least once a week if asthma recurred. The patient has had a history of asthma since she was a child. Based on family history, several other family members have a history of atopic allergies. Dental history reveals that the patient is currently undergoing fixed orthodontic treatment for the past 5 years. 
An extraoral examination showed no abnormalities. Intraoral examination of the dorsal tongue reveals a white plaque, easy to rub without leaving any red areas. Another finding in the middle of the tongue is a reddish area with a smooth surface, regular edges resembling the MRG, as shown in Fig. 1. The Oral Hygiene Index (OHI) was in the moderate category. The clinical diagnosis in this case, was confirmed as a coated tongue accompanied by suspect MRG. The treatments that have been given are instruction to brush the tongue using a tongue scraper, gargle using water shortly after the use of a steroid inhaler when the asthma attack subsides, administer the mouthwash containing povidone-iodine $1 \%$, and control 1 week later.
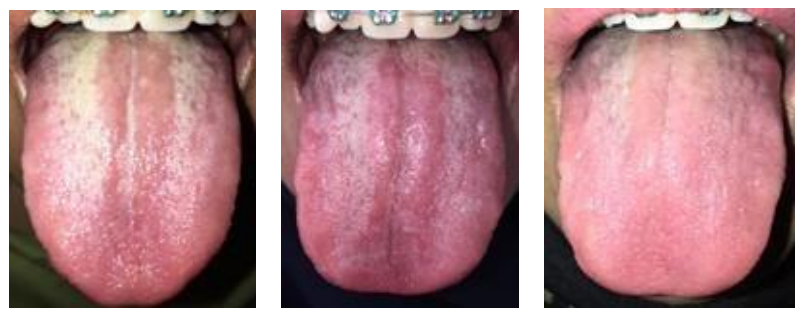

Fig.1: First Visi Fig.2 : Second Visit Fig.3 : Third Visit

The second visit, October $14^{\text {th }}, 2019$, was carried out 2 weeks later after the first visit. At this visit, the patient felt that her tongue was much more comfortable and the white color around the red area had started to thin, although the red area was still visible. A complaint of pain or itching is denied. From the first visit to the second visit, asthma attacks did not occur. Cleaning the tongue using a tongue scraper is still done 2 times a day. The intraoral examination showed yellowish-white areas on the right and left of the red area starting to thin (Fig. 2), which confirmed the clinical diagnosis as the coated tongue. Another finding on the median of the tongue as an area of redness, smooth surface, with regular edges was the same as at the first visit which was similar to MRG. The patient was instructed to keep their tongue clean at least 2 times a day and observed for 1 month.

At the third visit, about 1 month after the second visit, the patient felt that her tongue was getting better. The patient was reported in the last 1 month using a steroid inhaler several times. The patient always rinses her mouth with plain water after using a steroid inhaler, as recommended by the operator. Cleaning of the tongue with a tongue scraper was continued. Intraoral examination of the area of redness in the median dorsal tongue was persistent, with a smooth, irregular edge with a slightly yellowish-white surrounding area and was thinner than the second visit (Fig. 3). The sixweek case evaluation was quite favorable concerning white lesions on the tongue diagnosed as the coated tongue. In contrast, MRG-like red areas on the median dorsal tongue were another finding that was suspected as an oral manifestation of steroid inhaler use.

\section{DISCUSSION}

The tongue is an important part of the human body, which has a complex anatomy. The tongue can be considered as a mirror of oral and or systemic health. A dentist can be the first person to observe the lingual status and should be familiar with the different diagnoses of these conditions, for example, being able to link lingual abnormalities to a specific etiology [12]. In normal conditions, the tongue has a rough surface texture because the mucous membrane contains many lingual papillae, such as filiform, fungiform, foliate, and circumvallate papillae [9]. The unique structure of the dorsum papillary of the tongue supports the accumulation of debris and microorganisms that can contribute to changes in the surface texture of the tongue to be whiter, black, red, or yellowish. The formation of a lining of the tongue is a normal phenomenon, located mostly on the back and dorsal center of the tongue, tending to vary in color and thickness.

Changes in the surface texture of the tongue, especially on lingual papillae, can occur due to various etiologies. The tongue is continuously exposed to chemical, mechanical, and physical stimuli that, when too strong or chronic, may cause atrophic lesions [2][12]. In general, the normal tongue color is pink to slightly white and is often covered with a layer [16]. In this case report, a white coating that forms on the tongue is the main complaint, making the patient feel uncomfortable. The clinical appearance of the lesion as white plaque, easy to rub, and not easy to bleed is suspected as a white-coated tongue. A white-coated tongue is defined as a white coating on the surface of the tongue and visible adherence to the dorsum of the tongue. It is reported that this white layer consists of an accumulation of desquamated epithelial cells, metabolite blood cells, various types of food waste, leukocytes from periodontal pockets, and bacteria [16].

In other cases, the clinical appearance of a whitish tongue is oral thrush. Clinically the characteristics of oral thrush are white plaque, easy to rub, and leave a reddish area and bleed easily. The clinical diagnosis of oral thrush can not be established because the white lesions in this case, when rubbed, do not leave red areas and do not bleed easily. This is reinforced by the case evaluation after the first visit, where the white area on the dorsum of the tongue experienced significant changes. During the observation, the patient is advised to always brush the tongue using a tongue scraper at least twice a day. A study reported that the habit of regularly brushing the tongue has the potential to successfully reduce the coated tongue. Subjects who regularly brushed their tongues had a lower prevalence of coated tongue than those who did not brush their tongues regularly [17].

Another interesting finding, in this case, was an MRGlike area of depapillation in the median dorsum of the tongue, with a clinical appearance of asymptomatic reddish macules. The suspicion of MRG, in this case, was based on history, clinical examination, and evaluation of the case for several weeks in which the red macular appearance on the dorsum of the tongue did not change. Based on history, it is known that patients use steroid inhalers quite often to treat asthma attacks. One of the triggers for MRG is the use of a steroid inhaler. An inhaled steroid is a commonly used treatment in respiratory diseases manifesting with airway 
obstruction such as asthma and COPD [13]. It is known that prolonged use of a steroid inhaler with high frequency can reduce a person's resistance to infection. A steroid inhaler can suppress the individual's nonspecific inflammatory or T-cell-mediated immune response, causing a yeast infection. Several studies have shown that long-term use of inhaled steroids can alter the normal flora in the oral cavity and result in overgrowth of Candida sp. [11].

Median rhomboid glossitis is believed as a developmental disorder of tongue formation. The depapillated area is a persistent tuberculum impar that cannot fuse completely with lateral lingual swelling in the development of the tongue, resulting in a smooth, erythematous, and lacking papillae [18]. Today, however, most authors do not subscribe to the embryogenesis theory. Instead, they believe that the MRG is related to an infection of Candida albicans, which is the most common fungal organism of the oral cavity [11]. Apart from the clinical appearance, establishing the diagnosis of MRG, in this case, requires confirmation of laboratory examination. These tests include cytologic smears with potassium hydroxide, biopsy for periodic acidSchiff and Gram staining for tissue infiltration by spores and hyphae, or culture getting better. Besides, patients continue to carry out operator instructions to rinse with warm water after using steroid inhalers.

\section{CONCLUSION}

A steroid inhaler for the treatment of asthma can be associated with several lesions on the tongue, such as coated tongue and MRG. Confirmation of MRG by mycological examination is needed to ensure the presence of fungal colonization. In this case, although the appearance of the red lesion on the dorsal tongue resembles MRG, the diagnosis of MRG cannot be confirmed because the mycological examination has not been carried out.

\section{REFERENCES}

[1] D. Mirza, G. Raza, and Z.A. Abassi, "Median Rhomboid Glossitis: A Peculiar Tongue Pathology, Report of A Case And Review Of Literature," Int. J. Pharm. and Biol. Sci. vol. 6, Issue 4, pp. 51-53, 2016.

[2] M. Goregen, O. Miloglu, M. C. Buyukkurt, F. Caglayan, and A. E. Aktas, "Median Rhomboid Glossitis: A Clinical and Microbiological Study," Eur. J. Dent. vol. 5, pp. 367-372, 2011.

[3] J. Terai and M. Shimahara, "Atrophic tongue associated with candida," J. Oral. Pathol. Med. Vol. 34(7), pp. 397-400, September 2005.

[4] P. Panta and S.R. Erugula, "Median rhomboid glossitis developmental or candidal?," Pan. Afr. Med. J. Vol. 21, pp. 221, 2015. M. S. Shetty, U. M. Prabhu, K. Fernandez, P. Malli, F. A. Khan, P. Kumar and Rao, "Median Rhomboid Glossitis With Palatal 'Kissing Lesion'-A Case Report Of 12 Year Old Child," Int. J. Curr. Med. Pharm. Res. vol. 3, Issue 12, pp.27852786, 2017.
[5] V. der Wal, V. der Kwast and V. der Waal, "Median rhomboid glossitis: A followup study of 16 patients," J. Oral. Med. Vol. 41(2), pp. 117-120, 1986.

[6] N. S. Soysa and A.N. Ellepola, "The impact of cigarette/tobacco smoking on oral candidiasis: an overview," Oral. Dis. Vol. 11(5), pp. 268 - 273, September 2005.

[7] S. Bhattacharyya and B. Satarupa, "Fungal infections of the oral cavity," Int. J. Cur. Res. Rev. Vol. 5, pp. 83-88, 2013.

[8] I. Sanders and $\mathrm{Mu} \mathrm{L}$ "A 3-Dimensional Atlas of Human Tongue Muscles," Anat. Rec. (Hoboken), Vol. 296, No. 7, pp.1102-14, 2013.

[9] Moore KL, Dalley AF, Agur AMR, "Clinically oriented anatomy," 7th ed. Philadelphia: Lippincott Williams and Wilkins, 2013, pp. 936- 43.

[10] S. Patil, R. S. Rao, B. Majumdar, and S. Anil, "Clinical Appearance of Oral Candida Infection and Therapeutic Strategies," Front. microbiol. Vol. 6, pp.1391, 2015.

[11] M. Erriu, F.M.G. Pili, S. Cadoni, and V. Garau, "Diagnosis of Lingual Atrophic Conditions: Associations with Local and Systemic Factors. A Descriptive Review," Open. Dent. J. Vol. 10, pp. 619635, 2016.

[12] N. Godara, R. Godara, and M. Khullar, "Impact of inhalation therapy on oral health," Lung. India. Vol. 28, No. 4, pp. 272-275, 2011.

[13] K. Seerangaiyan, F. Juch and E.G. Winkel, "Tongue Coating: its characteristics and role in intra-oral halitosis and general health: A review," J. Breath. Res. Vol. 12(3), pp. 1752-7163, Mar 2018.

[14] M. Van Tornout, J. Dadamio, W. Coucke, and M. Quirynen, "Tongue coating: related factors," J. Clin. Periodontol. Vol. 40, No. 2, pp. 180-185, 2013.

[15] R. A. Omor, M. A. Arabeyat, A. N. Hiasat, M. S. Ajarmeh, and H.H.A. Fanas, "Prevalence and Factors Related to Tongue Coating among a Sample of Jordanian Royal Medical Services Dental Outpatients", JRMS. Vol. 22, No. 1, pp. 35-40, 2015.

[16] M. Goregen, O. Miloglu, M.C. Buyukkurt, F. Caglayan and A.E.Aktas, "Median Rhomboid Glossitis: A Clinical \& Microbiological Study," Eur. J. Dent. Vol. 5(4), pp. 367-372, Octoberr 2011. 